\title{
Preceding T-Cell-Mediated Rejection Is Associated with the Development of Chronic Active Antibody-Mediated Rejection by de Novo Donor-Specific Antibody
}

\author{
Takahiro Tsuji $^{\text {a Sari Iwasaki }}{ }^{a}$ Keishi Makita ${ }^{a}$ Teppei Imamoto ${ }^{a}$ \\ Naomichi Ishidate ${ }^{a}$ Akihiko Mitsuke $^{b}$ Nobuyuki Fukuzawa $^{b}$ \\ Hiroshi Haradab Yuichiro Fukazawa ${ }^{a}$ \\ aDepartment of Pathology, Sapporo City General Hospital, Sapporo, Japan; \\ bDepartment of Kidney Transplant Surgery, Sapporo City General Hospital, Sapporo, Japan
}

\section{Keywords \\ Acute T-cell-mediated rejection - Borderline changes . \\ Chronic active antibody-mediated rejection - de novo donor-specific antibody . Protocol biopsy}

\begin{abstract}
Aim: Chronic active antibody-mediated rejection (CAABMR) is an important cause of late-stage renal allograft loss. Early inflammatory events such as acute rejection and infection after transplantation are considered to be the risk factors of de novo donor-specific antibody (dnDSA) production. In this study, we investigated the relationship between predisposing T-cell-mediated rejection and dnDSA-positive CAABMR. Methods: We recruited 365 patients who underwent ABO-compatible renal transplantation at our hospital. Among them, 16 patients diagnosed as having dnDSA-positive CAABMR were designated as a CAABMR group, and 38 randomly selected patients were designated as a control group. All biopsies from 1 month after transplantation were included in the study. The presence or absence of borderline changes (BLCs), acute T-cell-mediated rejection (ATMR), microvascular inflammation (MVI), and $\mathrm{C} 4 \mathrm{~d}$ positive on peritubular capillaries (C4d-P) was examined. Results: In
\end{abstract}

the CAABMR group, BLC/ATMR was found in 12 cases (75\%), and the mean duration until appearance of BLC/ATMR was $282.7 \pm 328.7$ days. C4d-P was found in 11 cases (68.8\%), and the mean duration until its appearance was 1,432 $\pm 1,307$ days. MVI was found in all cases, and the mean duration until its appearance was $1,333 \pm 1,126$ days. The mean duration until diagnosis of CAABMR was 2,268 $\pm 1,191$ days. In the control group, BLC/ATMR was found in 13 cases (34.2\%), and the mean duration until the appearance of BLC/ATMR was $173.1 \pm 170.4$ days. C4d-P was found in 2 cases (5.3\%), and the durations until its appearance were 748 and 1,881 days. No cases of MVI were found in the control group. The frequency of BLC/ATMR was significantly higher in the CAABMR group $(p<0.01)$. Conclusion: Preceding BLC/ATMR is associated with the development of CAABMR with dnDSA.

(c) 2020 S. Karger AG, Basel

\section{Introduction}

Chronic active antibody-mediated rejection (CAABMR) is an important cause of late-stage renal allograft loss. CAABMR is characterized by chronic irreversible changes in the basement membrane of glomerular capillary loops

$\begin{aligned} & \text { karger@karger.com } \\ & \text { www.karger.com/nef }\end{aligned}$
Karger ${ }^{\prime /}$


and/or peritubular capillaries in addition to findings of acute tissue injury such as microvascular inflammation (MVI) and evidence of current antibody interaction with the vascular endothelium such as $\mathrm{C} 4 \mathrm{~d}$ staining in peritubular capillaries in the presence of donor-specific antibodies (DSAs). Both the presence of preformed DSA and the presence of de novo DSA (dnDSA) can result in the development of CAABMR, but CAABMR caused by dnDSA has a particularly poor outcome [1]. Early inflammatory events such as T-cell-mediated rejection (TCMR) and viral infection are suspected of being risk factors for dnDSA production in addition to a high degree of human leukocyte antigen mismatch, insufficient immunosuppression, and nonadherence $[2,3]$. Among them, a model in which early TCMR after transplantation triggers dnDSA production and subsequent progression to CAABMR has received attention in recent years [4-6]. The aim of this study was to clarify the relationship between TCMR and dnDSA-positive CAABMR by comprehensively reviewing biopsies including multiple protocol biopsies for CAABMR and control groups.

\section{Materials and Methods}

We recruited 365 patients who underwent $\mathrm{ABO}$-compatible renal transplantation at the Department of Kidney Transplant Surgery of our hospital from October 1987 to December 2013. Among them, 16 patients diagnosed as having dnDSA-positive CAABMR during the period from July 2010 to June 2015 were designated as a CAABMR group, and 38 randomly selected patients who did not develop CAABMR but for whom the number of biopsies was equivalent to that in the CAABMR group were designated as a control group. In our hospital, protocol biopsies are routinely performed in renal transplant patients at 1, 3, and 6 months after transplantation and then every 1 year after transplantation [7]. Therefore, data from protocol biopsies were dominant in this study (protocol biopsy/total biopsy $=366 / 395$ [92.7\%]). In the CAABMR group, biopsies from 1 month after transplantation to CAABMR diagnosis were included, and in the control group, biopsies from 1 month after transplantation to $7.2 \pm 3.4$ years were included. Two pathologists (TT and SI) reviewed all of the biopsy specimens for the presence or absence of borderline changes (BLC) and acute T-cell-mediated rejection (ATMR) as TCMR-related lesions. Also, data for when MVI appeared, when C4d became positive on peritubular capillaries (C4d-P), and when CAABMR was diagnosed were retrieved from pathology records. The positive thresholds of MVI and C4d were the thresholds according to Banff 2017, that is, $g+$ ptc $>1$ and C4d $>1$ by immunofluorescence [8]. Histological diagnoses were performed in accordance with the standards outlined at Banff 2017 [8]. Statistical analyses were performed with the $\chi^{2}$ test, Fisher's exact test, Student's $t$ test, Welch's $t$ test, and the Mann-Whitney's U test. This study was conducted with the approval of the Ethical Committee at Sapporo City General Hospital (H30-059-547).

\section{Results}

\section{Clinical Characteristics of the Patients}

Patient clinical characteristics are shown in Table 1. In the CAABMR group, an average of $7.8 \pm 2.6$ biopsies was performed until CAABMR diagnosis, and the diagnosis of CAABMR was made in $6.2 \pm 3.2$ years after transplantation. In the control group, an average of $7.1 \pm 1.7$ biopsies was performed during the follow-up period of $7.2 \pm 3.4$ years. The number of episode biopsies was significantly larger, and the latest serum Cr level and latest urinary protein level were significantly higher in the CAABMR group. There were 2 cases of nonadherence in the CAABMR group, but there were no cases in the control group. There were 2 cases of cytomegalovirus (CMV) infection in the CAABMR group and 3 cases of CMV infection in the control group. One case of CMV infection in the control group was complicated with bacterial infection. Of the cases diagnosed with BLC/ATMR in the CAABMR group, 2 were treated with steroid pulse therapy (mPSL) and 4 were treated with mPSL and 15-deoxyspergualin (DSG). Of the cases diagnosed with BLC/ATMR in the control group, 1 case was treated with mPSL and 2 cases were treated with $\mathrm{mPSL}$ and DSG. There was no significant difference between the 2 groups in the frequency of nonadherence and the frequency of infection $(p=0.08$ and $p=$ 0.62 ). The number of cases treated with BLC/ATMR tended to be greater in the CAABMR group than in the control group ( $p=0.054)$, but there was no difference between the 2 groups in the treatment method of $\operatorname{BLC} / \operatorname{ATMR}(p=1.0)$.

\section{Frequencies of BLC/ATMR in CAABMR and Control Groups}

The frequencies of BLC/ATMR are summarized in Figure 1. In the CAABMR group, BLC was observed in 8 cases, and ATMR was observed in 4 cases (grade I: 3 cases, grade II: 1 case). In the control group, 10 cases of BLC and 3 cases of ATMR grade I were observed. Therefore, BLC/ATMR was found in 12 cases (75\%) in the CAABMR group and in 13 cases (34.2\%) in the control group. Thus, the frequency of BLC/ATMR was significantly higher in the CAABMR group ( $\chi^{2}$ test, $p<0.01$ ). The average numbers of biopsies judged as BLC/ATMR per case were $1.8 \pm 1.6$ in the CAABMR group and $0.1 \pm 0.3$ in the control group, which were significantlylarger in the CAABMR group $(p<0.005)$ (Table 1$)$.

In the CAABMR group, only 2 cases of ATMR (grades IA and IIB) were detected by episode biopsy, and 10 $(83.3 \%)$ of the 12 cases were detected by protocol biopsies. In the control group, only 1 case of ATMR (grade IB)
14

Nephron 2020;144(suppl 1):13-17 DOI: $10.1159 / 000512659$
Tsuji et al. 
Table 1. Patient characteristics

\begin{tabular}{llll}
\hline & $\begin{array}{l}\text { CAABMR group, } \\
n=16\end{array}$ & $\begin{array}{l}\text { Control group, } \\
n=38\end{array}$ & $p$ value \\
\hline Recipient age, years & $39.9 \pm 15.5$ & $40.4 \pm 15.9$ & $\mathrm{~ns}$ \\
Recipient sex (male/female) & $10 / 6$ & $26 / 12$ & $\mathrm{~ns}$ \\
ABO-compatible & 16 & 38 & - \\
Living/deceased & $13 / 3$ & $30 / 8$ & $\mathrm{~ns}$ \\
Type of dnDSA (class I/II/I + II) & $3 / 7 / 6$ & na & ns \\
HLA mismatches & $2.8 \pm 1.8$ & $2.2 \pm 1.9$ & $\mathrm{~ns}$ \\
Number of total biopsies (times) & $7.8 \pm 2.6$ & $7.1 \pm 1.7$ & $\mathrm{~ns}$ \\
Number of protocol biopsies (times) & $6.3 \pm 2.2$ & $7.0 \pm 1.7$ & $<0.001$ \\
Number of episode biopsies (times) & $1.5 \pm 1.8$ & $0.1 \pm 0.3$ & $<0.005$ \\
Number of biopsies determined & & & $\mathrm{ns}$ \\
$\quad$ to be BLC/ATMR & $1.8 \pm 1.6$ & $0.1 \pm 0.3$ & 0.04 \\
Most recent biopsy, years & $6.2 \pm 3.2$ & $7.2 \pm 3.4$ & 0.01 \\
Most recent serum Cr, mg/dL & $1.4 \pm 0.5$ & $1.1 \pm 0.3$ & $\mathrm{~ns}$ \\
Most recent proteinuria, g/g Cr & $1.2 \pm 2.3$ & $0.4 \pm 0.6$ & $\mathrm{~ns}$ \\
Nonadherence & 2 & 0 & $\mathrm{CMV}: 3$ \\
Infection & CMV:2 & $($ with bacterial infection:1) & ns \\
Treatment for BLC/ATMR & mPSL + DSG:4, & mPSL + DSG:3, & mPSL:1 \\
\hline
\end{tabular}

ATMR, acute T-cell-mediated rejection; BLC, borderline change; CAABMR, chronic active antibody-mediated rejection; CMV, cytomegalovirus; dnDSA, de novo donor-specific antibody; DSG, 15-deoxyspergualin; HLA, human leukocyte antigen; mPSL, methylprednisolone pulse therapy; na, not applicable; Cr, creatinine.

was detected by episode biopsy, and $12(92.3 \%)$ of the 13 cases were detected by protocol biopsies.

\section{Onset Timings of Histological Lesions Associated with} TCMR and ABMR in CAABMR and Control Groups

The timings of onset of various lesions (BLC/ATMR, C4d-P, MVI, and CAABMR) are shown in Figure 2 and Table 2. Figure 2a shows lesions in the CAABMR group. The mean duration until the appearance of BLC/ATMR was $282.7 \pm 328.7$ days (15-1,100 days). C4d-P was found in 11 cases $(68.8 \%)$, and the mean duration until the appearance of C4d-P was $1,432 \pm 1,307$ days (201-3,354 days). MVI was found in all cases, and the mean duration until the appearance of MVI was 1,333 $\pm 1,126$ days (194,332 days). The mean duration until diagnosis of CAABMR was 2,268 $\pm 1,191$ days (558-4,332 days). The lesions in the control group are shown in Figure 2b. The mean duration until the appearance of BLC/ATMR was $173.1 \pm 170.4$ days (14-594 days). C4d-P was found in 2 cases $(5.3 \%)$, and the durations until the appearance of C4d-P were 748 and 1,881 days. No case of MVI was found in the control group. There was no significant difference between the durations until the appearance of BLC/ATMR in the CAABMR group and the control group $(p=0.51)$.

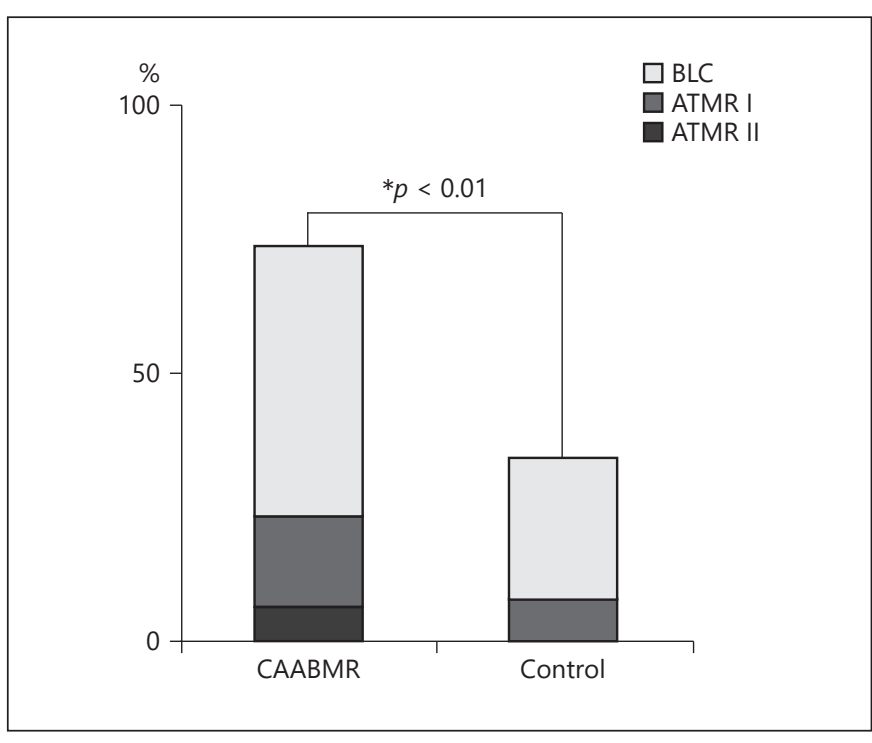

Fig. 1. Comparison of the frequencies of BLCs and acute T-cellmediated rejection (ATMR) in the CAABMR group and the control group. BLC/ATMR was found in 12 cases $(75 \%)$ in the CAABMR group and in 13 cases $(34.2 \%)$ in the control group. Thus, the frequency of BLC/ATMR was significantly higher in the CAABMR group $\left({ }^{*} \chi^{2}\right.$ test, $\left.p<0.01\right)$. CAABMR, chronic active antibody-mediated rejection; BLC, borderline change; ATMR, acute T-cell-mediated rejection. 
Table 2. Comparison of the frequencies and the timings of onset of various lesions

\begin{tabular}{lll}
\hline & CAABMR group, $n=16$ & Control group, $n=38$ \\
\hline BLC/ATMR & $n=12(75 \%), 282.7 \pm 328.7(15-1,100)$ days & $n=13(34.2 \%), 173.1 \pm 170.4(14-594)$ days \\
C4d-P & $n=11(68.8 \%), 1,432 \pm 1,307(558-4,332)$ days & $n=2(5.3 \%), 748$ and 1,881 days \\
MVI & $n=16(100 \%), 1,333 \pm 1,126(19-4,332)$ days & $n=0$ \\
CAABMR & $n=16(100 \%), 2,268 \pm 1,191(558-4,332)$ days & $n=0$ \\
\hline
\end{tabular}

ATMR, acute T-cell-mediated rejection; BLC, borderline change; C4d-P, C4d positive on peritubular capillaries; CAABMR, chronic active antibody-mediated rejection; MVI, microvascular inflammation.

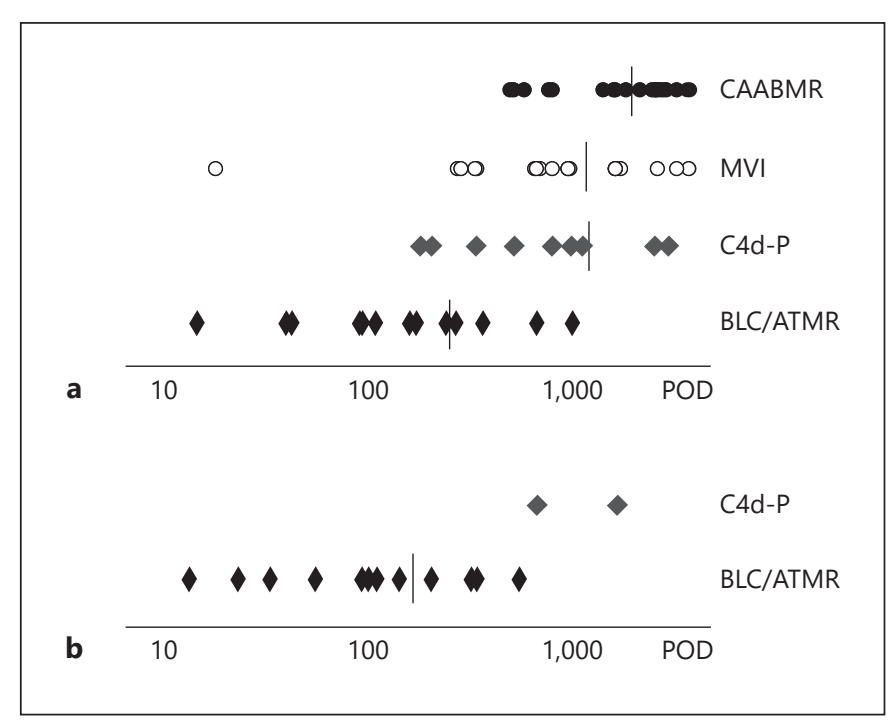

Fig. 2. Comparison of the timing of onset of various lesions in the CAABMR group (a) and the control group (b). The dates when lesions BLC/ATMR, C4d-P, MVI, and CAABMR were first detected are plotted in the graph. Black bars indicate average values. The $x$-axis is on a logarithmic scale. POD, postoperative day; CAABMR, chronic active antibody-mediated rejection; BLC, borderline change; ATMR, acute T-cell-mediated rejection; C4d-P, C4d positive on peritubular capillaries; MVI, microvascular inflammation.

\section{Discussion}

In this study, by reviewing multiple biopsy records of $\mathrm{ABO}$-compatible kidney transplantation, the incidence and onset time of TCMR-related lesions and CAABMRrelated lesions were clarified. The appearance order and appearance timing of these lesions were consistent with the CAABMR evolution model proposed by Wiebe et al. [6].

The frequency of BLC/ATMR in the CAABMR group was significantly higher than that in the control group. This result is consistent with the results of studies showing that ATMR is a risk factor for CAABMR $[5,6,9]$. In the CAABMR group, preceding BLC/ATMR may be a trigger for dnDSA production. The majority $(83.3 \%)$ of BLC/ATMR cases found in the CAABMR group were subclinical cases, and they were detected by protocol biopsies, suggesting that frequent protocol biopsies are useful for detection of BLC/ATMR. Since subclinical BLC is often overlooked by pathologists, it is important for pathologists to carry out detailed examinations in order to diagnose early BLC/ATMR after transplantation and promptly link them to treatment in order to suppress DSA production.

There are several limitations in this study. First, this study was a retrospective study. Second, although C4d staining was carried out for all biopsies, including biopsies in the control group, the DSA test was not carried out in many of the cases in the control group, and the presence or absence of a DSA was, therefore, not confirmed in all cases. Third, immunohistochemistry of the SV40-T antigen was carried out in only some cases, and it was, therefore, not possible to completely exclude the possibility of polyomavirus infection. However, since none of the cases showed characteristic histology of polyomavirus infection or an increase in the number of urinary decoy cells, the influence of polyomavirus infection is considered to be limited.

In summary, the frequency of BLC/ATMR in the CAABMR group was significantly higher than that in the control group, suggesting that preceding BLC/ATMR may be a trigger for dnDSA production. Appropriate diagnosis and treatment of early BLC/ATMR are thought to be important for suppressing dnDSA production and subsequent progression to CAABMR.

\section{Acknowledgement}

We wish to thank Mr. Masaki Kutsumura for assistance with pathology record retrieval.
16

Nephron 2020;144(suppl 1):13-17 DOI: $10.1159 / 000512659$
Tsuji et al. 


\section{Statement of Ethics}

This study was conducted with the approval of the Ethical Committee at Sapporo City General Hospital (H30-059-547).

\section{Conflict of Interest Statement}

All authors have no competing interests to declare.

\section{References}

1 Aubert O, Loupy A, Hidalgo L, Duong van Huyen JP, Higgins S, Viglietti D, et al. Antibody-mediated rejection due to preexisting versus De Novo Donor-specific antibodies in kidney allograft recipients. J Am Soc Nephrol. 2017;28(6):1912-23.

2 Zhang R. Donor-specific antibodies in kidney transplant recipients. Clin J Am Soc Nephrol. 2018;13(1):182-92.

3 Dieplinger G, Everly MJ, Briley KP, Haisch CE, Bolin P, Maldonado AQ, et al. Onset and progression of de novo donor-specific antihuman leukocyte antigen antibodies after BK polyomavirus and preemptive immunosuppression reduction. Transpl Infect Dis. 2015; 17(6):848-58
4 Haas $\mathrm{M}$. The relationship between pathologic lesions of active and chronic antibody-mediated rejection in renal allografts. Am J Transplant. 2018;18(12):2849-56.

5 Moreso F, Carrera M, Goma M, Hueso M, Sellares J, Martorell J, et al. Early subclinical rejection as a risk factor for late chronic humoral rejection. Transplantation. 2012;93(1):41-6.

6 Wiebe C, Gibson IW, Blydt-Hansen TD, Karpinski M, Ho J, Storsley LJ, et al. Evolution and clinical pathologic correlations of de novo donor-specific HLA antibody post kidney transplant. Am J Transplant. 2012;12(5):1157-67.

7 Tsuji T, Yanai M, Itami H, Ishii Y, Akimoto M, Fukuzawa N, et al. Microvascular inflammation in early protocol biopsies of renal allografts in cases of chronic active antibodymediated rejection. Nephrology. 2015; 20(Suppl 2):26-30.
8 Haas M, Loupy A, Lefaucheur C, Roufosse C, Glotz D, Seron D, et al. The Banff 2017 Kidney Meeting Report: revised diagnostic criteria for chronic active $\mathrm{T}$ cell-mediated rejection, antibody-mediated rejection, and prospects for integrative endpoints for next-generation clinical trials. Am J Transplant. 2018;18(2): 293-307.

9 El Ters M, Grande JP, Keddis MT, Rodrigo E, Chopra B, Dean PG, et al. Kidney allograft survival after acute rejection, the value of follow-up biopsies. Am J Transplant. 2013; 13(9):2334-41. 